\title{
EvoDock: Optimization of protein-ligand binding interfaces
}

\author{
M. Edich, M. Friedrichs \\ Bioinformatics Department at Bielefeld University \\ medich@PHYSnet.uni-hamburg.de
}

Evolution has led to proteins being able to specifically bind molecules. They have evolved to bind a great variety of chemical substances, from carbohydrates to small organic molecules as well as ions or other macro molecules. This power of evolution can also be harnessed in silico, using applications like Rosetta, to engineer proteins so that they bind ligands with higher specificity [1].

However, these bioinformatical algorithms are limited as they often cannot take all aspects of molecular modeling, like protein flexibility, solvent simulation, or energy calculation, reliably into account [2-4]. In addition, most complex applications are usually difficult to use for novices.

Here, we present EvoDock, a modular and easy-to-use pipeline which is capable of integrating multiple molecular modeling programs into an evolutionary algorithm, to predict optimized variants of ligand-binding proteins.

Furthermore, we demonstrate how its predictions could be confirmed by crystallographic structures and will discuss the potential usage of binding energy and the energy of the overall structure to determine a protein's fitness.

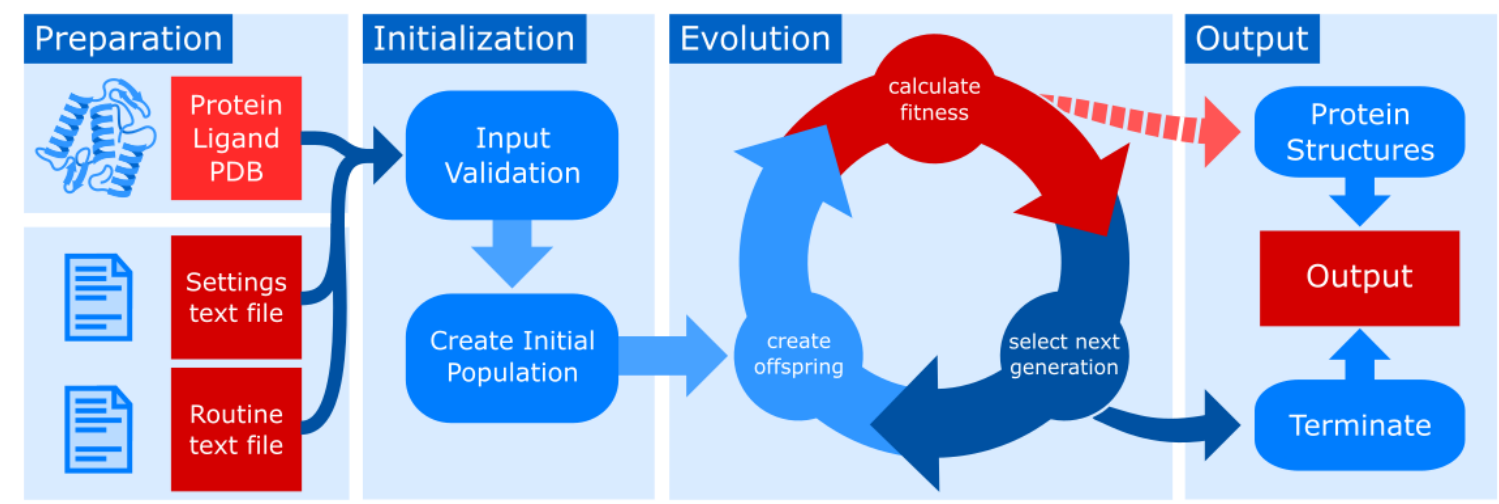

Figure 1. Workflow of EvoDock. Only the preparation is performed manually, which is followed by an initialization. During the evolution, numerous mutational variants from the input protein are created and evaluated, leading to new protein variants until one of the termination criteria is met. The molecular modeling programs are integrated into a pipeline within the fitness calculation.

[1] Thyme, S., \& Baker, D. (2014). Redesigning the specificity of protein-DNA interactions with Rosetta. In Homing Endonucleases (pp. 265-282). Humana Press, Totowa, NJ.

[2] Spyrakis, F., BidonChanal, A., Barril, X., \& Javier Luque, F. (2011). Protein flexibility and ligand recognition: challenges for molecular modeling. Current topics in medicinal chemistry, 11(2), 192-210.

[3] Pavlovicz, R. E., Park, H., \& DiMaio, F. (2020). Efficient consideration of coordinated water molecules improves computational protein-protein and protein-ligand docking discrimination. PLoS computational biology, 16(9), e1008103.

[4] Aldeghi, M., Gapsys, V., \& de Groot, B. L. (2018). Accurate estimation of ligand binding affinity changes upon protein mutation. ACS central science, 4(12), 1708-1718.

Keywords: protein engineering; evolutionary algorithms; molecular modeling; Rosetta; structural bioinformatics

We thank Prof. Dr. Kristian Müller and Lennard Karsten as well as Cassandra Königs and Prof. Dr. Ralf Hofestädt (all from Bielefeld University) for the insightful discussions. 